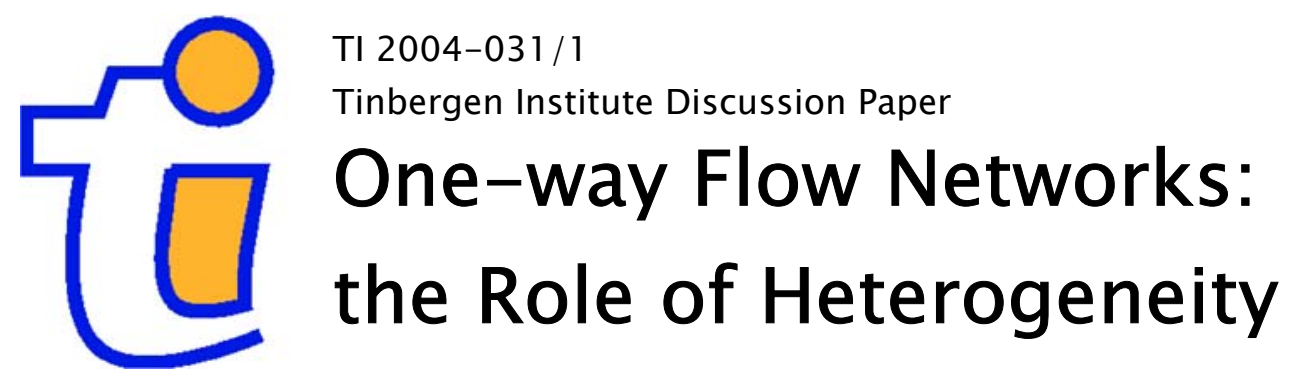

Andrea Galeotti

Econometric Institute, Faculty of Economics, Erasmus Universiteit Rotterdam, and Tinbergen Institute. 


\section{Tinbergen Institute}

The Tinbergen Institute is the institute for economic research of the Erasmus Universiteit Rotterdam, Universiteit van Amsterdam, and Vrije Universiteit Amsterdam.

Tinbergen Institute Amsterdam

Roetersstraat 31

1018 WB Amsterdam

The Netherlands

Tel.: $\quad+31(0) 205513500$

Fax: $\quad+31(0) 205513555$

Tinbergen Institute Rotterdam

Burg. Oudlaan 50

3062 PA Rotterdam

The Netherlands

Tel.: $\quad+31(0) 104088900$

Fax: $\quad+31(0) 104089031$

Please send questions and/or remarks of nonscientific nature to driessen@tinbergen.nl.

Most TI discussion papers can be downloaded at http://www.tinbergen.nl. 


\title{
One-way flow networks: the role of heterogeneity*
}

\author{
Andrea Galeotti ${ }^{\dagger}$
}

March 2004

\begin{abstract}
I study a one-way flow connections model in which players are heterogeneous with respect to values and the costs of establishing a link. I first show that values and costs asymmetries are crucial in determining the level of connectedness of a network. Interestingly, unconnected equilibria are asymmetric and central players may emerge. Second, I show that non-singleton components have a wheel architecture as far as the costs of linking are not partner specific. Otherwise also the flower architecture constitutes an equilibrium. I interpret these results as saying that symmetric connections across players are a peculiar feature of homogenous environments; by contrast, in heterogeneous settings players hold asymmetric connections which are accompanied by the emergence of central players.
\end{abstract}

*I thank Sanjeev Goyal, Alessandro Lanteri, Caterina Marchionni, J.L. Moraga-Gonzalez, Alex Konovalov and Rob Van der Noll for useful comments. I also acknowledge the participants of the Nake Day 2003 in Amsterdam.

†Tinbergen Institute \& Econometric Institute, Erasmus University Rotterdam. E-mail: galeotti@few.eur.nl 


\section{Introduction}

The role of social and economic networks in shaping individual behavior and aggregate phenomena has been widely documented in recent years. ${ }^{1}$ This provides the main motivation for developing a theory which aims to understand how networks form and their architectural properties. The most popular model of network formation is the connections model. ${ }^{2}$ Variants of this model have been proposed in order to analyse different social and economic situations. Nevertheless, much of the work has explored settings with homogeneous players. However, ex-ante asymmetries across players arise quite naturally in reality. For instance, in the context of information networks it is often the case that some individuals are more interested in particular issues and therefore better informed, which makes them more valuable contacts. Similarly, individuals differ in communication and social skills and it seems natural that forming links is cheaper for some individuals as compared to others. ${ }^{3}$ In this paper, I analyse the role played by ex-ante asymmetries across players in shaping network architectures. To do this, I consider a version of the connections model where individuals invest in social ties unilaterally (one-sided network) and the flow of benefits is frictionless and directed only towards the investor (one-way flow network).

Bala and Goyal (2000) analyse this model with homogenous players (costs of forming links and values of accessing players are homogenous) and they show that if a player's payoffs are increasing in the number of other players accessed and decreasing in the number of links formed, a strict Nash network is either a wheel (a connected network in which each player creates and receives one link) or the empty network (with no links). The intuition for this result is as follows. Consider a minimally connected network where player 1 initiates a link with player 2 and 3 , and each of these players has a link with player 1 . Whenever players are homogeneous, this network is not a strict equilibrium: player 2 is indifferent between maintaining the link with 1 and switching to player 3, instead. A generalization of this argument implies that a connected strict equilibrium is symmetric and has a wheel architecture. It is worth noting that this result depends crucially on the assumption of homogenous values and costs. To see this, assume that player 1 is just slightly cheaper to be linked with than player 2 and 3 , ceteris paribus. This small introduction of heterogeneity implies that the network described in the example above becomes a strict equilibrium. In the current paper, I study the role played by heterogeneous players in shaping equilibrium networks.

\footnotetext{
${ }^{1}$ There is a large body of work on this subject. See e.g., Burt (1992) on careers of professional managers, Montgomery (1991) on wage inequality in labour markets, Granovetter (1974) on the flow of job information, and Coleman (1966) on diffusion of medical drugs.

${ }^{2}$ This model has been extensively studied in the literature; see e.g., Bala and Goyal (2000a, 2000b), Dutta and Jackson (2000), Goyal (1993), Jackson and Wolinsky (1996), and Watts (2001a, 2001b).

${ }^{3}$ In other settings players can be classified in term of their cost of being accessed. For example, on the web the terminology user-friedly web site is used to describe home pages which are easier to access as compared to others.
} 
Players are heterogeneous in terms of the costs of linking and the values of accessing other players.

I start with a setting where values and costs of linking are heterogeneous across players but the heterogeneity is not partner specific: the cost for player $i$ to invest in a social tie is $c_{i}$, and the benefit to player $i$ to access another player is $V_{i}$. I show that a connected equilibrium is a wheel network and that an unconnected equilibrium network is either a center-sponsored star, a wheel with singletons, a wheel with local center-sponsored stars or empty (Proposition 3.1). Figure 1 illustrates all strict equilibria in a society with four players. This result shows that players' heterogeneity alters the level of connectedness of equilibrium networks. In any non-empty unconnected equilibrium there is a set of players sharing a maximum amount of information while the remaining players are socially isolated (they do not access any information). In sharp contrast with the homogeneous setting these equilibria are asymmetric and central players may emerge: $(i)$ the players maximally informed are connected in a wheel component and the players socially isolated are either $\left(i i_{a}\right)$ singletons or $\left(i i_{b}\right)$ spokes of centersponsored stars. I finally note that the wheel is robust to asymmetries which are independent from the potential partner.

I then turn to settings where heterogeneity also depends on the potential partner. I show that the wheel architecture is still prominent if costs are not partner specific; otherwise any minimally connected network is a strict equilibrium for some costs and values (Proposition 3.2). This leads me to conclude that costs heterogeneity is responsible for shaping the architecture of equilibrium networks.

To investigate the role heterogeneity plays in shaping the architecture of equilibrium networks I impose some restriction on the cost of forming links. This leads me to study a targeted-partner model: the cost of forming a link with a player $i$ is symmetric across players, but each player has a different cost of being accessed. I show that a connected equilibrium is either a wheel or a flower, in which case the player with the lowest access cost occupies the central position. Furthermore, an unconnected strict equilibrium is either a wheel with a local periphery-sponsored star or a flower with a local periphery-sponsored star (Proposition 4.1). ${ }^{4}$ Figure 2 illustrates these architectures in a society composed of 4 players.

I now comment on three aspects of this result. First, the unique asymmetric connected equilibrium has a flower architecture and the center of the flower is the player with the lowest access cost. The center is the only player in the network which promotes and receives more than one link and his function is to connect sets of players which would be otherwise disconnected. Second, unconnected strict equilibria have well defined architectures. A set of individuals shares information with each other (the core group),

\footnotetext{
${ }^{4}$ The strict equilibria with only singleton components are a limit case of the architectures decribed in Proposition 4.1 and they are described in the appendix (Proposition 4.2).
} 
while the remaining players (the periphery group) access the information of the core group directly from the players with the lowest access cost in the population. I finally note that the flower (and its variants) is a less efficient equilibrium as compared to the wheel equilibrium (and its variants). The inefficiency inherent in asymmetric equilibria arises from the over-investment by the central player.

This paper is a contribution to the theory of network formation. This is a very active area of research currently (see references in footnote 2). Most of the existing literature focuses on homogeneous player models. My analysis elaborates on the respective roles of values and costs of forming links heterogeneity in shaping equilibrium architectures in a one-way flow connections model. The work that comes closest to mine is Kim and Wong (2003). They study a one-sided connections model with heterogeneous players where agents form two-flow connections but basic links are only one-flow. In other words, this implies that a player $i$ accesses player $j$ only if there exists a sequence of basic links connecting $i$ to $j$ and vice versa. My work departs from Kim and Wong (2003) in two directions. First, I analyse different form of players' asymmetries, while Kim and Wong (2003) focus exclusively on settings where asymmetries are not partner specific. Second, I do not distinguish between basic links and flow connections, which implies that in my framework a player can access another individual, without the reverse being necessarily true. ${ }^{5}$

Finally, I relate my findings to a recent experimental paper by Falk and Kosfeld (2003). This paper shows that the predictions based on Nash and Strict Nash equilibria for the one-way flow model are consistent with the experimental results, while they generally fail in the two-way flow model. ${ }^{6}$ The authors argue that the success of the one-way flow model relies, among other things, on the strategic symmetry (symmetric distribution of links) which characterizes equilibrium networks under the one-way flow assumption. The analysis developed in the current paper shows that the property of symmetric distribution of links depends on the assumption of homogeneous players. An experiment which takes into account ex-ante asymmetries in the costs of forming links may help to understand the role played by strategic symmetry in the formation process of a network.

The rest of the paper is organized as follows. Section 2 introduces the model. Section 3 presents the results on equilibrium networks under general cost and value heterogeneity. Section 4 analyses the targeted-partner model. Section 5 concludes. Proofs are provided in the Appendix.

\footnotetext{
${ }^{5}$ For example, the web is characterized by one-way link and one-way flow connections.

${ }^{6}$ Bala and Goyal (2000) shows that with homogeneous players and when information flow is bidirectional a strict equilibrium is either a center-sponsored star (only one player, the center, promotes all the links) or empty (no links).
} 


\section{The Model}

Let $N=\{1, \ldots, n\}$ be a set of players and let $i$ and $j$ be typical members of this set. We shall assume throughout that the number of players is $n \geq 3$. Each player is assumed to possess some information of value to himself and to other players. He can augment his information by communicating with other people; this communication takes resources, time and effort and is made possible via pair-wise links.

A strategy of player $i \in N$ is a (row) vector $g_{i}=\left(g_{i, 1}, \ldots, g_{i, i-1}, g_{i, i+1}, \ldots, g_{i, n}\right)$ where $g_{i, j} \in\{0,1\}$ for each $j \in N \backslash\{i\}$. We say that player $i$ has a link with $j$ if $g_{i, j}=1$. We assume throughout the paper that a link between $i$ and $j$ allows player $i$ to access $j$ 's information. The set of strategies of player $i$ is denoted by $\mathcal{G}_{i}$. Throughout the paper we restrict our attention to pure strategies. Since player $i$ has the option of forming or not forming a link with each of the remaining $n-1$ players, the number of strategies of player $i$ is clearly $\left|\mathcal{G}_{i}\right|=2^{n-1}$. The set $\mathcal{G}=\mathcal{G}_{1} \times \ldots \times \mathcal{G}_{n}$ is the space of pure strategies of all the players.

A strategy profile $g=\left(g_{1}, \ldots, g_{n}\right)$ in $\mathcal{G}$ can be represented as a directed network. Let $g \in \mathcal{G}$, I say that there is a path in $g$ from $i$ to $j$ if either $g_{i, j}=1$ or there exist players $j_{1}, \ldots, j_{m}$ distinct from each other and $i$ and $j$ such that $\left\{g_{i, j_{1}}=\ldots=g_{j_{m}, j}=1\right\}$. We write $i \stackrel{g}{\rightarrow} j$ to indicate a path from $i$ to $j$ in $g$. Given two players $i$ and $j$ in $g$, the geodesic distance, $d_{i, j}(g)$, is defined as the length of the shortest path from $i$ to $j$. Furthermore, we define $N^{d}(i ; g)=\left\{k \in N \mid g_{i, k}=1\right\}$ as the set of players with whom $i$ maintains a link while we refer to $N(i ; g)=\{k \in N \mid i \stackrel{g}{\rightarrow} k\} \cup\{i\}$ as the set of players that $i$ observes in $g$. Let $\mu_{i}^{d}: \mathcal{G} \rightarrow\{1, \ldots, n\}$ and $\mu_{i}: \mathcal{G} \rightarrow\{1, \ldots, n\}$ be defined as $\mu_{i}^{d}(g)=\left|N^{d}(i ; g)\right|$ and $\mu_{i}(g)=|N(i ; \bar{g})|$.

Given a network $g$, a non-singleton component of $g$ is a non-singleton set $C(g) \subset N$ where $\forall i, j \in C(g)$ there exists a path between them and there is not a path between $\forall i \in C(g)$ and a player $k \in N \backslash C(g)$. A component $C(g)$ of a network $g$ is minimal if $C(g)$ is no longer a component upon replacement of a link $g_{i, j}=1$ between two agents $i, j \in C(g)$ by $g_{i, j}=0$, ceteris paribus. A network $g$ is minimal if every component of $g$ is minimal. A network $g$ is connected if it has a unique component containing all players. If the unique component is minimal the network $g$ is minimally connected. A network which is not connected is unconnected. Given a network $g$, a player $i$ is a singleton player if $g_{i, j}=g_{j, i}=0$ for any $j \in N$. Finally, the empty network, denoted as to $g^{e}$, is an unconnected network where no links are formed.

To complete the definition of a normal-form game of network formation, we specify the payoffs. Let $V_{i, j}$ denote the benefits to player $i$ from accessing player $j$. Similarly, let $c_{i, j}$ denote the cost for player $i$ of forming a link with player $j$. The payoff to player $i$ in a network $g$ can be written as follows: 


$$
\Pi_{i}(g)=\sum_{j \in N(i ; g)} V_{i, j}-\sum_{j \in N^{d}(i ; g)} c_{i, j}
$$

We shall assume that $c_{i, j}>0$ and $V_{i, j}>0$ for all $i, j \in N .^{7}$

Given a network $g \in \mathcal{G}$, let $g_{-i}$ denote the network obtained when all of player $i$ 's links are removed. Note that the network $g_{-i}$ can be regarded as the strategy profile where $i$ chooses not to form a link with anyone. The network $g$ can be written as $g=g_{i} \otimes g_{-i}$ where the ' $\otimes$ ' indicates that $g$ is formed as the union of the links in $g_{i}$ and $g_{-i}$. The strategy $g_{i}$ is said to be a best response of player $i$ to $g_{-i}$ if:

$$
\Pi_{i}\left(g_{i} \otimes g_{-i}\right) \geqslant \Pi_{i}\left(g_{i}^{\prime} \otimes g_{-i}\right) \text { for all } g_{i}^{\prime} \in \mathcal{G}_{i} .
$$

The set of all of player $i^{\prime} s$ best responses to $g_{-i}$ is denoted by $\mathcal{B R}_{i}\left(g_{-i}\right)$. Furthermore, a network $g=\left(g_{1}, \ldots, g_{n}\right)$ is said to be a Nash network if $g_{i} \in \mathcal{B} \mathcal{R}_{i}\left(g_{-i}\right)$ for each $i$, i.e. players are playing a Nash equilibrium. If a player has multiple best responses to the equilibrium strategies of the other players then this could make the network less stable as the player can switch to a payoff equivalent strategy. This switching possibility in non-strict Nash networks has been exploited and has been shown to be important in refining the set of equilibrium networks in earlier work (see e.g., Bala and Goyal (2000)). So we will focus on strict Nash equilibria in the present paper. A strict Nash equilibrium is a Nash equilibrium where each player gets a strictly higher payoff from his current strategy than he would with any other alternative strategy.

\section{General Heterogeneity}

In this section I investigate the effects of values and costs of linking heterogeneity on the level of connectedness and the architecture of strict equilibria. I shall show that values and costs heterogeneity are equally important in determining the level of connectedness of equilibrium networks, while only costs heterogeneity shapes the architecture of non-singleton components. I shall also show that when players are heterogeneous, equilibrium networks are asymmetric.

I start with a consideration of a setting in which each player has a distinct cost of linking as well as a distinct benefit of accessing other players. While these costs and values vary across players, they are independent from the identity of the partner, i.e. $V_{i, j}=V_{i}$ and $c_{i, j}=c_{i}$, for any $i, j \in N$. For example, some individuals are more expert in surfing the web as compared to others; this allows them to access other internet

\footnotetext{
${ }^{7}$ The results developed further qualitatively carry on when relaxing the linearity assumption of the payoffs functions.
} 
members to a lower cost, ceteris paribus. ${ }^{8}$ I first introduce some architectures which will prove useful in the analysis.

A star architecture is an unconnected network where there exists a player $i$, the center, such that either $g_{i, j}=1$ or $g_{j, i}=1$ for any $j \in N \backslash\{i\}$, and no other links are formed. The network $g$ has a center-sponsored star architecture if $g$ is a star and the center forms all the links. A non-singleton component has a wheel architecture if players within the component are arranged as $\left\{i_{1}, \ldots, i_{n}\right\}$ with $g_{i_{2}, i_{1}}=\ldots=g_{i_{n}, i_{n-1}}=g_{i_{1}, i_{n}}$ and there are no other links between players within the component. A wheel architecture is a connected network with the unique component being a wheel. A wheel network with local center-sponsored stars is an unconnected network with a unique wheel component, say $C(g)$, and where $\forall j \notin C(g), \exists ! i \in C(g)$ such that $g_{i, j}=1$. Finally, a wheel network with singleton players is an unconnected network with a unique wheel component composed of at least three players and where $g_{i, j}=g_{j, i}=0$ for any $i \notin C(g)$ and for any $j \in N$.

The next result shows which networks can be sustained in equilibrium.

Proposition 3.1: Let payoffs satisfy (1) and assume that $c_{i, j}=c_{i}$ and $V_{i, j}=V_{i}$ $\forall j \in N \backslash\{i\}$. A connected strict equilibrium is a wheel. Otherwise, a strict equilibrium is either the empty network, the wheel with singletons, the wheel with local centersponsored stars or the center-sponsored star. Conversely, any of such network is a strict equilibrium for some $\left\{c_{i}, V_{i}\right\}_{i \in N}$.

Figure 1 illustrates all strict equilibria in a society composed of 4 players. I represent a link $g_{i, j}=1$ as an edge starting at $j$ with the arrowhead pointing at $i$.

The proof of Proposition 3.1 proceeds as a sequence of Lemmas. I sketch here the main steps. I first show that a strict Nash network is minimal. This follows from the nodecay assumption. Secondly, using a standard switching argument I show that each player receives at most one link (Lemma 3.1). Third, using this equilibrium property it follows that each non-singleton component has a wheel architecture (Lemma 3.2). Therefore, a connected strict equilibrium is a wheel. Fourth, I take up the case of non-empty unconnected equilibria in which each component is composed of a single player. Using the finiteness of the set of players I show that an equilibrium is a center-sponsored star network (Lemma 3.3). Finally, an elaboration of the arguments used in the previous lemmas establishes the result for unconnected equilibria which have at least a non-singleton component.

Proposition 3.1 provides some interesting insights. As in the homogenous setting, the unique connected equilibrium is the wheel. Therefore, the wheel architecture is

\footnotetext{
${ }^{8}$ Similarly, some individuals may value more information provided on the web as compared to others. In general, individuals differ in communication and social skills and it seems natural that the costs of establishing links as well as the values of accessing informations vary across individuals.
} 
prominent also in settings where costs and values asymmetries are partner independent. Next, values and costs heterogeneity alters the level of connectedness of strict equilibria. In any unconnected (and non-empty) equilibrium there is a set of players accessing a maximum amount of information while all the other players are socially isolated (they do not access any information). Furthermore, the maximally informed players are connected in a wheel, while the isolated players are either singletons or spokes of center-sponsored stars. Thus, unconnected equilibria are generally asymmetric and central players may emerge. Finally, I note that the results presented in Proposition 3.1 carry on in settings with homogenous values (costs of linking) and heterogeneous costs of linking (values). This implies that as far as heterogeneity is independent from the partner, costs and values asymmetries have equivalent effects on strategic interaction.

I now ask under which conditions ex-ante asymmetries across players alter the architecture of equilibrium networks. The next result establishes that the wheel architecture is prominent as far as the costs of linking are partner independent.

Proposition 3.2: Let payoffs satisfy (1). First, assume $c_{i, j}=c_{i}$ while values vary freely, then a connected equilibrium is a wheel. Conversely, the wheel is an equilibrium for some $\left\{c_{i}, V_{i, j}\right\}_{i, j \in N}$. Second, assume $V_{i, j}=V_{i}$ while costs vary freely, then a connected equilibrium is minimal; conversely, any minimally connected network is a strict equilibrium for some $\left\{c_{i, j}, V_{i}\right\}_{i, j \in N}$.

I note that when values vary freely while costs asymmetries are partner independent, connected equilibria have (still) a wheel architecture. This result also holds with homogenous costs of linking. Differently, when the costs of linking are allowed to vary freely across players, the only restriction imposed by the equilibrium notion to connected network is minimality. This results holds regardless of values asymmetries.

The analysis of this section can be summarized as follows. First, the level of connectivity of a network is equally sensitive both to values and costs heterogeneity. Interestingly, unconnected equilibria are asymmetric and central players may emerge. Second, asymmetries in values do not alter the architecture of non-singleton components as compared to the homogeneous setting. The same observation applies when costs heterogeneity is independent from the potential partner. Third, when the costs of linking are allowed to be partner specific, social interaction leads to a 'everything is possible' type of result. Hence, I can conclude that it is costs of linking heterogeneity which is mainly responsible in shaping the architecture of equilibrium networks. The next section explores the possibilities to set plausible conditions on the cost parameters in order to obtain further restrictions on the architecture of equilibrium networks. 


\section{Targeted-Partner Model}

In this section I consider a setting where values are homogeneous, while the costs of linking are exclusively partner specific. In particular, each player $i$ has a different cost of being accessed, which is however homogenous with respect to the players who initiate a link with $i$. For example, some web sites are more user-friendly than others and this feature allows players to access the information provided there more easily. ${ }^{9}$

Formally, let $V_{i, j}=V$, for any $i, j \in N$ and assume the following costs structure:

$$
c_{i, j}=c_{j} \text { for any } i \in N
$$

I shall assume that $c_{1}>0$ and, without loss of generality, that $c_{j}<c_{x}$ whenever $j<x .^{10}$

Given a network $g$ and (3), the payoff to player $i$ can be rewritten as follow:

$$
\Pi_{i}(g)=\mu_{i} V-\sum_{j \in N^{d}(i ; g)} c_{j}
$$

I introduce some additional notation. An unconnected network with a unique wheel component, say $C(g)$, and where $g_{j, i}=1$ for any $j \notin C(g)$ and a unique player $i \in C(g)$ is called a wheel with a local periphery-sponsored star with center $i$. A flower component, $C(g)$, of a network $g$ partitions the set of players belonging to $C(g)$ into a central player, say $i$, and a collection of $\mathcal{P}=\left\{P_{1}, \ldots, P_{q}\right\}$, where each $P \in \mathcal{P}$ is non-empty. A set $P$ of agents is referred to as a petal. Denote the agents in $P$ as $\left\{j_{1}, \ldots, j_{n}\right\}$. A flower component is then defined by setting $\left\{g_{i, j_{1}}=g_{j_{1}, j_{2}}=\ldots=g_{j_{n}, i}=1\right\}$ for any $P \in \mathcal{P}$ and $g_{i, j}=0$ otherwise. If $g$ is connected with a flower component and $i$ is the center, then $g$ is a flower network with center $i$. An unconnected network with a unique flower component where player $i$ is the center and where $g_{j, i}=1$ for any $j \notin C(g)$ is called a flower with a local periphery-sponsored star with center $i$.

Figure 2 depicts all the aforementioned possible architectures in a society composed of four players.

\footnotetext{
${ }^{9}$ I provide some other examples. Individuals have different opportunity costs. It seems natural to consider that to access players with higher opportunity costs is more costly as compared to others. To apply for some jobs is less costly than for others. Different countries have different immigration policies. Countries which implement more strict immigration policy are more difficult to be accessed as compared to countries with "soft" immigration policies.

${ }^{10}$ Further, I shall discuss the implication of allowing groups of players having the same accessability cost.
} 


\subsection{Strict Equilibrium Networks}

I start by introducing some necessary conditions to characterize strict Nash equilibria. The role of these conditions is to constrain the arrangement of players belonging to the architectures I have introduced above. I start defining the ordered condition for a wheel network with a local periphery sponsored star.

Definition 1. A wheel with a local periphery-sponsored star network, say g, where player $h$ is the center, is ordered if for any $g_{i, j}=1, i, j \in C(g)$ then $(i) c_{j}-c_{y}<$ $d_{j, y}(C(g)) V$ for any $y \in C(g)$ and $(i i) c_{j}-c_{y}<d_{j, h}(C(g)) V$ for any $y \notin C(g)$.

Condition $(i)$ requires that any player $i$ linked with a player $j$ in a wheel component does not find it profitable to switch to another player belonging to the same wheel. Condition (ii) takes care of the switching possibilities of a player belonging to a component with players outside the component. I note that a wheel network is ordered if condition $(i)$ in Definition 1 is satisfied. I now turn to define the ordered condition for networks with a flower component.

Definition 2. A flower with a local periphery sponsored star network, say g, where player $h$ is the center, is ordered if for any $P \in \mathcal{P}$ and for any $g_{i, j}=1, i, j \in P$, then (i) $c_{j}-c_{y}<d_{j, y}(P(g)) V, \forall y \in P$ and $(i i) c_{j}-c_{y^{\prime}}<d_{j, h}(P(g)) V, \forall y^{\prime} \notin P$.

The interpretation of Definition 2 is analogous to the one of Definition 1. I note that as $c_{n}-c_{1}<V$, the ordered conditions in definition (1) and (2) are always satisfied.

The next proposition provides the set of strict equilibria in this model. I focus on equilibria in which there exists at least one non-singleton component. ${ }^{11}$

Proposition 4.1: Let (3)-(4) be satisfied. A connected strict equilibrium is either an ordered wheel or an ordered flower where player 1 is the center. An unconnected strict equilibrium with at least a non-singleton component is either an ordered wheel or an ordered flower with a local periphery sponsored star where player 1 is the center. Conversely any such network is a strict equilibrium for some $\left\{c_{i}, V\right\}_{i \in N}$.

The proof of Proposition 4.1 proceeds as a sequence of Lemmas. I sketch here the main steps. First, the assumption of no-decay in the information flow implies that a strict equilibrium is minimal. Second, using a standard switching argument I show that in a non-singleton component only the player with the lowest access cost can receive more than one link (Lemma 4.1). This implies that a connected equilibrium is either a wheel or a flower where player 1 is the center (Lemma 4.2). Third, I show that if a player $j \notin C(g)$ promotes a link with a player $i \in C(g)$, then $j$ does not

\footnotetext{
${ }^{11}$ The analysis of equilibria in which each component is composed of a single player is provided in the appendix (see proposition 4.2). The architecture of these equilibria are a limit case of the architectures of equilibria with a non-singleton component.
} 
receive any link. Suppose player $j^{\prime}$ forms a link with $j$, then the player linked with $i$ in $C(g)$ gains by switching to player $j$ (Lemma 4.3). Finally, using the fact that the set of players is finite I show that players within a component do not promote links with players outside that component (Lemma 4.4). Lemma 4.3 and 4.4 together imply that any two players belonging to two different components access two distinct sets of agents. A simple switching argument establishes that in a strict equilibrium at most one non-singleton component exists.

I discuss some aspect of this result. The first remark is about the flower architecture. When players are heterogenous in terms of their costs of being accessed, asymmetric connected networks are strategically viable and a coordinator emerges: the player with the lowest access cost. The coordinator connects sets of players who would otherwise be disconnected. It is worth noting that the flower network arises also in homogeneous settings when a small amount of decay in the information flow is introduced. In particular, Bala and Goyal (2000) show that for a sufficiently small amount of decay the flower architecture is the only strict Nash network. ${ }^{12}$ In that case the role of the center is to decrease the distance between players. Differently, in this model the flower arises because the center is the more profitable player to be linked with. It is clear that the result on decay of Bala and Goyal (2000) is reinforced in our setting because the advantage of linking with the center is not only having short information channels but also the decrease in investment cost.

The second remark is about unconnected equilibria. In these equilibria players can be divided in two groups: one, a core group composed of players belonging to a non-singleton component and two, a periphery group composed of all the remaining players. Interestingly, players within the core group are accessed by all players in the society; by contrast, no player accesses the individuals belonging to the periphery group.

Third, I remark on the assumption that the ranking of players in term of their costs of being accessed is strictly increasing. ${ }^{13}$ Suppose that players in the society can be grouped in $m$ distinct and different groups, $N=\cup_{i=1}^{m} N_{i}$ and that players belonging to the same group have the same cost of being accessed. If $N_{1}$ is composed of a single player, then an ordered flower network where player 1 is the center can be sustained as a strict equilibrium. Indeed, in this case the ordered conditions are enough to take care of switching possibilities. By contrast, when also group $N_{1}$ is composed of more than one player the problem becomes more delicate. The following example clarifies this point. Consider three groups, say 1,2 and 3, each composed of three players, say $a, b$ and $c$. Let $g$ be the network depicted in figure 3 and let $c_{3}<(n-1) V$. The network $g$ is not strict Nash: player $2_{a}\left(3_{a}\right)$ is indifferent between retaining a link

\footnotetext{
${ }^{12}$ See Bala and Goyal (2000) for a detailed discussion on this issue.

${ }^{13} \mathrm{In}$ other words, I discuss the effect of allowing groups of players to have the same accessibility cost.
} 
with $1_{a}$ or switching either to $1_{b}$ or $1_{c}$; differently all other players have a unique best response. However, it has to be noted that the best responses of every player are insensitive to such changes by player $2_{a}$ and $3_{a}$. This implies that this network $g$ along with the possible best responses of player $2_{a}$ and $3_{a}$ constitutes a minimal curb set of the game. ${ }^{14}$ Using standard results on best response dynamics it follows that this set is absorbing, meaning that once a best response dynamic enters this set it will cycle within this set forever.

Finally, I compare the efficiency property of the wheel architecture with respect to the flower architecture. ${ }^{15}$ Consider a wheel and a flower network, it is easy to see that both architectures generate the same amount of network externalities, but the former requires a lower level of total investment. ${ }^{16}$ Thus, the flower network is less efficient as compared to the wheel because the center in the flower over-invest in social ties. The same argument holds along all the variants of the flower and wheel architecture. This remark implies that if we move from an homogenous setting towards an heterogeneous environment in a way that social interaction becomes less costly, then strategic interaction leads to the emergence of inefficient equilibria. For example, let's assume that players are fully homogeneous and $V>c$. In this case the unique equilibrium is the wheel architecture; furthermore, the wheel is uniquely efficient. Assume now that a player becomes slightly cheaper to be linked with as compared to the others. This small introduction of heterogeneity implies that the flower architecture is also an equilibrium. However, this equilibrium is inefficient.

\section{Discussions}

I have studied a connections model where heterogeneous players decide unilaterally to invest in social ties which leads a direct return only to the investor. The main results can be summarized as follows. First, the level of connectedness of a network is equally sensitive to values and costs heterogeneity. Furthermore, non-empty unconnected equilibria have an asymmetric distribution of links and central players may emerge in equilibrium. Second, the wheel architecture (along with its variants) is robust to players' asymmetries as far as costs heterogeneity is independent from the potential partner. By contrast, when the costs of linking are allowed to vary freely the only restriction imposed by strategic interaction on the architecture of non-singleton components is minimality. I explore the role played by costs asymmetries which are partner specific in the targeted-partner model: each player has a distinct cost of be-

\footnotetext{
${ }^{14}$ More precisely the set defined is a supertight curb set, which is a generalization of the strict Nash notion. For more detail see Basu and Weibull (1991) and Galeotti, Goyal and Kamphorst (2003).

${ }^{15}$ I consider the social welfare of a network $g$ as the sum of payoffs of all players.

${ }^{16}$ It is easy to see that the wheel architecture is the more efficient architeture in the class of connected architectures.
} 
ing accessed. Here, non-singleton components are either wheel or flower. The flower network has an asymmetric architecture where the central player connects sets of players who would otherwise be disconnected. I finally note that asymmetric architectures are less efficient than symmetric ones. I interpret these result as saying that symmetric connections across players is a peculiar feature of homogenous environments; by contrast, in heterogeneous settings players hold asymmetric connections, accompanied by the emergence of central players.

\section{Appendix}

\section{Proof of Proposition 3.1.}

First part. I first note that an equilibrium network is minimal. This follows from the assumption of no-decay in the information flow. The proof now proceeds as a sequence of Lemmas. The next result shows that in equilibrium each player receives at most one link.

Lemma 3.1: Let $g$ be a strict equilibrium. If $g_{i, j}=1$ then $g_{k, j}=0$ for any $k \in N \backslash\{i\}$.

Proof: Suppose, for a contradiction that $g_{i, j}=1$ and $g_{k, j}=1$. Since $g$ is minimal, $i$ does not access player $k$ in $g$; however, in this case player $i$ strictly prefers to delete the link with player $j$ and linking up with player $k$, instead. This is a contradiction.

Using this result I show that each non-singleton component part of a strict equilibrium is a wheel

Lemma 3.2: Let $C(g)$ be a non-singleton component part of a strict equilibrium $g$. Then $C(g)$ has a wheel architecture.

Proof: I note that if a player $i$ belongs to a non-singleton component, say $C(g)$, then $g_{i, j}=1$ for at least one player $j \in C(g)$ and $g_{k, i}=1$ for at least one player $k \in C(g)$. These two observations and Lemma 3.1 imply that each player $i \in C(g)$ receives one and only one link from the players belonging to $C(g)$. I now claim that for any player $i \in C(g)$, then $g_{i, j}=1$ for only one player $j \in C(g)$. Suppose, for a contradiction, that for some $i \in C(g), g_{i, j}=g_{i, k}=1$ for some $j, k \in C(g)$ and $j \neq k$. Since $j, k \in C(g)$ then $j$ and $k$ must access player $i$; therefore, there exist two paths $\left\{g_{i, k}=g_{k, k_{1}}=\ldots g_{k_{n-1}, k_{n}}=g_{k_{n}, i}=1\right\}$ and $\left\{g_{i, j}=g_{j, j_{1}}=\ldots g_{j_{n-1}, j_{n}}=g_{j_{n}, i}=1\right\}$. Since $i$ receives only one link it must be the case that $j_{n}=k_{n}$. However, the same argument applies for player $j_{n}\left(=k_{n}\right)$, and therefore it must be the case that $j_{n-1}=k_{n-1}$. By induction, it follows that $k_{1}=j_{1}$; since $k \neq j$, it follows that $k_{1}\left(=j_{1}\right)$ must receive more than one link. This constitutes a contradiction. These observations altogether implies that $C(g)$ is minimally connected and it has a symmetric architecture. It 
is readily seen that the unique directed graph which satisfies these properties is the wheel. This proves the Lemma.

Lemma 3.1 and 3.2 implies that a connected equilibrium network is a wheel. I now take up the case of non-empty unconnected strict equilibria in which each component is a singleton. The next Lemma proves the result.

Lemma 3.3: A non-empty unconnected strict equilibrium where each component is a singleton has a center-sponsored star architecture.

Proof: Since $g$ is non-empty there exists some $g_{i, j}=1$. There are two cases. First, suppose $g_{j, j^{\prime}}=0$ for any $j^{\prime} \in N$. Since $g$ is strict Nash it must hold that $V_{i}-c_{i}>0$; this implies that $\exists i \stackrel{g}{\rightarrow} j^{\prime}$ for any $j^{\prime} \in N$. Select player $k$ which is at the maximum distance from $i$ in $g$, i.e. $k=\arg \max _{j^{\prime} \in N} d_{i, j^{\prime}}(g)$. If $d_{i, k}(g)=1$ player $i$ accesses each other player directly and the proof trivially follows. If $d_{i, k}(g)>1$, it must be the case that $\left\{g_{i, j_{1}}=g_{j_{1}, j_{2}}=\ldots=g_{j_{m}, k}=1\right\}$ and $g_{k, s}=0$ for any $s \in N$. Since $g$ is strict Nash then $V_{j_{m}}-c_{j_{m}}>0$ and therefore player $j_{m}$ accesses any player in $g$. This implies that player $i$ and $j_{m}$ belongs to a non-singleton component, which constitutes a contradiction. Second, suppose $g_{j, j^{\prime}}=1$ for some $j^{\prime} \in N$. Since $g$ has only singleton components it follows $j^{\prime} \in N \backslash\{i\}$. Therefore, if $g_{j \prime, k}=0$ for any $k \in N$, the previous argument applies and we end-up with a contradiction. If $g_{j^{\prime}, k}=1$ for some $k$, then it must be the case that $k \in N \backslash\{i, j\}$. Since the number of players is finite, it must exist a player $h$ which is accessed by player $i$ via the link $g_{i, j}=1$ and such that $g_{h, h^{\prime}}=1$ and $g_{h^{\prime}, h^{\prime \prime}}=0$ for any $h^{\prime \prime} \in N$. However, also in this case the fact that $g$ is strict Nash implies that $V_{h}-c_{h}>0$ and therefore player $h$ must access player $i$ in $g$. This constitutes a contradiction. Hence the proof follows.

I now turn to unconnected strict equilibria where at least a non-singleton component exists. Let $C_{1}(g), C_{2}(g), \ldots, C_{m}(g)$ be the components of an unconnected strict equilibrium $g$. Lemma 3.1 and 3.2 implies that: (a) $C_{x}(g)$ is a wheel $\forall x=1, \ldots, m$; (b) $g_{j, i}=0, \forall i \in C_{x}(g)$ and $\forall j \in N \backslash\left\{C_{x}(g)\right\}, \forall x \in\{1, \ldots, m\}$.

Lemma 3.4: Let $g$ be a strict equilibrium and let $i \in C_{x}(g)$. If $g_{i, j}=1$ where $j \notin \cup_{y=1}^{m} C_{y}(g)$, then $g_{j, k}=0$ for any $k \in N$.

Proof: Suppose not, i.e. $g_{i, j}=g_{j, k}=1$. Lemma 3.1 implies that $k \notin \cup_{y=1}^{m} C_{y}(g) \cup\{j\}$; moreover, it also implies that if $g_{k, h}=1$ then $h \notin \cup_{y=1}^{m} C_{y}(g) \cup\{j, k\}$. Suppose that $g_{k, h}=0$ for any $h \notin \cup_{y=1}^{m} C_{y}(g) \cup\{j, k\}$; since $g$ is a strict Nash it follows that $V_{j}>c_{j}$. In this case player $j$ strictly gains by forming a link with player $i$. This constitutes a contradiction. If $g_{k, h}=1$ for some $h \notin \cup_{y=1}^{m} C_{y}(g) \cup\{j, k\}$ we can iterate the argument above and since the number of players is finite the proof follows.

Lemma 3.1. 3.3 and 3.4 implies that any pair of players, say $i$ and $j$, belonging to two different components, say $C_{x}(g)$ and $C_{y}(g)$, access two distinct set of players, 
i.e. if $i \in C_{x}(g)$ and $j \in C_{y}(g)$, with $x \neq y$, then $N_{i}(g) \cap N_{j}(g)=\Phi$. The next Lemma uses this observation to prove that a strict equilibrium network has at most one non-singleton component.

Lemma 3.5: A strict equilibrium has at most one non-singleton component.

Proof: Suppose not and let, without loss of generality, $\left|N_{i}(g)\right| \geq\left|N_{j}(g)\right|$, where $i \in C_{x}(g)$ and $j \in C_{y}(g)$, and $x \neq y$. Since $g$ is strict Nash it follows that $\left|N_{j}(g)\right| V_{j}-$ $c_{j}>0$; however, if this is the case, player $j$ is weakly better off by deleting his link in $C_{y}(g)$ and linking up with player $i$, i.e. $\left|N_{i}(g)\right| V_{j}-c_{j} \geq\left|N_{j}(g)\right| V_{j}-c_{j}>0$. This contradiction proves the lemma.

The next lemma completes the analyses of unconnected strict equilibria which have a non-singleton component.

Lemma 3.6: Let $g$ an unconnected strict equilibrium with a non-singleton components. Then $g_{j, j \prime}=0$ for any $j, j^{\prime} \notin C(g)$.

Proof: Suppose, for a contradiction, that $g_{j, j^{\prime}}=1$. Lemma 3.1 implies that each player outside the component does not access players belonging to the component. Therefore, Lemma 3.5 applies to the set of players $N \backslash\{C(g)\}$, i.e. $g_{j, j^{\prime}}=1$ for some $j^{\prime} \notin C(g)$. However, in this case player $j$ strictly gains by creating a link with a player $i \in C(g)$. This constitutes a contradiction. Hence, Lemma 3.5 follows.

The combination of Lemma 3.2, 3.4, 3.5 and 3.6 implies that an unconnected strict equilibrium with some non-singleton components is either a wheel with local centersponsored stars, a wheel with singleton players or a wheel with some local centersponsored star and some singleton player. It is immediate to see that this last architecture cannot be sustained as a strict equilibrium. This completes the proof of the first part of the proposition.

Second part. First, let $g$ be the empty network and let $c_{i}>V_{i}$ for any $i \in N$; it follows that $g$ is a strict equilibrium. Second, let $g$ be a wheel and set $c_{i}<V_{i}$ for any $i \in N$; it follows that $g$ is a strict equilibrium. Third, let $g$ be a center-sponsored star network where player $i$ is the center. For any $j \in N \backslash\{i\}$ let $c_{j}>(n-1) V_{j}$, while for the central player $i$ let $c_{i}<V_{i}$. It follows that $g$ is a strict equilibrium. Fourth, let $g$ be a wheel network with singleton players and let $C(g)$ be the wheel component in $g$. For any player $i \in C(g)$ set $V_{i}$ and $c_{i}$ such that $c_{i} \in\left(V_{i},(|C(g)|-1) V_{i}\right)$, while for any other player $j \notin C(g)$ set $V_{j}$ and $c_{j}$ such that $c_{j}>|C(g)| V_{j}$. It follows that $g$ is a strict equilibrium. Finally, let $g$ be a wheel network with local center-sponsored stars and let $C(g)$ be the wheel component. For any player $i \in C(g)$, set $c_{i}$ and $V_{i}$ such that $c_{i}<V_{i}$ and for any other player $j \notin C(g)$ set $c_{j}$ and $V_{j}$ such that $c_{j}>(n-1) V_{j}$. It follows that, $g$ is a strict equilibrium. This completes the proof of the second part. 
The proof of Proposition 3.1 is completed.

Proof of Proposition 3.2: I start by assuming that $c_{i, j}=c_{i}$, for any $j \in N$, while $V_{i, j}$ varies freely. Let $g$ be a connected strict equilibrium network. The nodecay assumption implies that $g$ is minimal. Furthermore, Lemma 3.1 and 3.2 of Proposition 3.1 applies also to this case; hence $g$ has a wheel architecture. I now prove the converse. Let $g$ be a wheel network and for any link $g_{i, j}=1$ let $V_{i, j}>c_{i}$. This implies that each player $i$ finds it optimal to maintain his links and not to form any other links. Hence, the first part of the proof follows.

I now turn to the second case, i.e. $V_{i, j}=V_{i}$ for any $j \in N$ and $c_{i, j}$ varies freely. Let $g$ be a connected network. The no-decay assumption implies that $g$ is minimal. Conversely, let $g$ be a minimally connected network. For any link $g_{i, j}=1$ let $c_{i, j}<I_{i, j} V_{i}$, while for any $g_{i, j}=0$ let $c_{i, j}>(n-1) V$. These two conditions assure that each player is playing his unique best response. This completes the proof of the Proposition.

\section{Proof of Proposition 4.1.}

First part. I first note that the no-decay assumption implies that an equilibrium network is minimal. The proof now proceeds as a sequence of Lemmas. The first lemma shows that if a player belonging to a non-singleton component receives more than one link, then this player has the lowest access cost across players within that component.

Lemma 4.1: Let $C(g)$ be a non-singleton component of a strict equilibrium and let $j \in C(g)$. If $g_{i, j}=1$, for more that one player $i \in C(g)$ then $c_{j}=\min _{j^{\prime} \in C(g)} c_{j^{\prime}}$.

Proof: Suppose, for a contradiction, that $g_{i, j}=g_{k, j}=1$, for some $i, k \in C(g), i \neq k$ and $c_{h}<c_{j}$ for some $h \in C(g) \backslash\{j\}$. Since $g$ is minimal and $i, k, j \in C(g)$, player $i$ (or $k$ ) accesses $h$ via the link $g_{i, j}=1$ (or $g_{k, j}=1$ ). In this case, player $i$ (or $k$ ) strictly gains by deleting the link with $j$ and linking up with $h$, instead. This contradiction proves the lemma.

Lemma 4.2 A non-singleton component of a strict equilibrium is either a wheel or a flower.

Proof: We have two possibilities. First, suppose any player $i \in C(g)$ receives at most one link in $C(g)$. I note that Lemma 3.2 in Proposition 3.1 also applies in this case. Therefore $C(g)$ has a wheel architecture. Second, suppose $i \in C(g)$ receives more than one link, i.e. $g_{j_{1}, i}=g_{j_{2}, i}=\ldots=g_{j_{k}, i}=1$. Lemma 4.1 implies that player $i$ is the player with lowest access cost in $C(g)$. I now claim that if player $i$ receives $k$ distinct links, then $C(g)$ is a flower with $k$ petals. Since $\left\{i, j_{1}, \ldots, j_{k}\right\} \in C(g)$, there exists a path $i \rightarrow j_{x}$, for any $x=1, \ldots, k$. The same argument presented in Lemma 3.2 of Proposition 3.1 implies that if player $h$ belongs to the path $i \rightarrow j_{x}$ then $h$ cannot 
belong to another path $i \rightarrow j_{y}, x \neq y$ and $x, y=1, \ldots, k$. Consider an arbitrary path $i \rightarrow j_{x}$; using Lemma 3.2, it follows that each player belonging to that path forms one and only one link. It is readily seen that the only possibility left is that $C(g)$ has a flower architecture with $k$ petals and player $i$ is the center. This completes the proof.

Lemma 4.2 implies that a connected strict equilibrium is either a wheel or a flower with player $j=1$ the center. I now note that if $g$ has wheel architecture and it is strict Nash then the wheel is ordered. Suppose, for a contradiction, that $g$ is a wheel but it is not ordered, i.e. for some $g_{i, j}=1, i, j \in C(g)$, then $c_{j}-c_{y} \geq d_{j, y}(C(g)) V$ for some $y \in C(g)$. If this is the case, player $i$ (weakly) gains by deleting the link with player $j$ and linking up with $y$, instead. A similar argument shows that a flower equilibrium network is ordered.

I now turn to consider unconnected networks with some non-singleton components.

Lemma 4.3: Let $g$ be an unconnected network and let $C(g)$ be a non-singleton component. If $g_{k, i}=1$, for some $i \in C(g)$ and $k \notin C(g)$ then $g_{k^{\prime}, k}=0$ for any $k^{\prime} \in N$.

Proof: Assume, for a contradiction, that $g_{k^{\prime}, k}=1$ for some $k^{\prime}$. I first note that $k^{\prime} \notin C(g)$; for otherwise $k \in C(g)$. Then, let us assume that $k^{\prime} \notin C(g)$ and let player $i^{\prime} \in C(g)$ be linked with player $i$, i.e. $g_{i^{\prime}, i}=1$. I note that if player $i^{\prime}$ deletes the link with $i$ and creates a new link with player $k$, he will still observe all the players he was accessing before the switching (via the new link with player $k$ ) and in addition he accesses all players that $k$ accesses in $g$ and that are not accessed by $i$ in $g$, i.e $\tilde{\sigma}_{k}=|\{h: \nexists i \stackrel{g}{\rightarrow} h \wedge \exists k \stackrel{g}{\rightarrow} h\}|$. Since $g$ is strict Nash it must be the case that $c_{k}-c_{i}>\tilde{\sigma}_{k}$. Next, I note that if player $k^{\prime}$ deletes the link with player $k$ and creates a new link with player $i$, then he (player $k^{\prime}$ ) will not accessed anymore all players $h$ that player $i$ does not access in $g$ and that $k^{\prime}$ accesses in $g$ exclusively via the link with the player $k$, i.e $\sigma_{k}=\left|\left\{h: \nexists i \stackrel{g}{\rightarrow} h \wedge \exists k \stackrel{g}{\rightarrow} h \wedge \nexists k^{\prime} \stackrel{g^{\prime}}{\rightarrow} h\right\}\right|$. Since $g$ is strict Nash it must be the case that $c_{k}-c_{i}<\sigma_{k}$. It is readily seen that $\sigma_{k} \leq \tilde{\sigma}_{k}$; however, this implies that the two conditions are incompatible. This contradiction completes the proof of the lemma.

Lemma 4.4: Let $g$ be a strict equilibrium and let $C_{1}(g), \ldots, C_{m}(g)$ be the non-singleton components belonging to $g$. If $i \in C_{x}(g)$, then $g_{i, j}=0, \forall j \notin C_{x}(g)$.

Proof: For a contradiction assume that $i \in C_{x}(g)$ and that $g_{i, j}=1$ for some $j \notin$ $C_{x}(g)$. Moreover, let player $i^{\prime} \in C_{x}(g)$ and such that $g_{i, i^{\prime}}=1$. Lemma 4.3 implies that $j \notin \cup_{y=1}^{m} C_{y}(g)$. Therefore, we have two possibilities. First, assume that $\forall k \in N$, $g_{j, k}=0$. In this case player $j$ does not access any player. Since player $i$ has a link with player $i^{\prime}$ and $g$ is strict Nash then player $j$ strictly gains to initiate a link with player $i^{\prime}$. This is a contradiction. Second, assume that $g_{j, k}=1$ for some $k \in N$. Lemma 4.3 
implies that $k \notin \cup_{y=1}^{m} C_{y}(g) \cup\{j\}$. Iterating the previous argument it follows that if $g_{k, h}=0$ for any $h \in N$, then player $k$ strictly prefers to create a link with player $i^{\prime}$. Therefore, it must be the case that $g_{k, h}=1$ for some $h$, which, using Lemma 4.3, implies that $h \notin \cup_{y=1}^{m} C_{y}(g) \cup\{j, k\} \forall y=1, \ldots, m$. We can continue to iterate this argument and since the set of players is finite, it must be the case that there exists a player, say $l$, who does not access any player and that is accessed by player $i$ via a path in $g$. However, in this case player $l$ strictly prefers to create a link with player $i^{\prime}$. Thus the proof follows.

Lemma 4.4 implies that if $i \in C_{x}(g)$ and $j \in C_{y}(g), x \neq y \forall x, y=1, \ldots, m$, then $N_{i}(g) \cap N_{j}(g)=\Phi$. Using this property we note that only one non-singleton component can be part of a strict equilibrium. For otherwise, one player belonging to a non-singleton component (weakly) gains be switching to another non-singleton component. Summarizing, it follows that a strict equilibrium with at least a non-singleton components must satisfy: (1) there exists a unique non-singleton component, say $C(g)$, which has a wheel architecture or a flower architecture, and (2) each player within the non-singleton component does not promote links with player outside the component (Lemma 4.4). We now note that each player outside the non-singleton component, say $j \notin C(g)$, accesses players within the components. This fact and Lemma 4.4 implies also that each player outside the component is directly linked with the player who has the lowest access cost within the component. It is now immediate to see that the player with the lowest access cost in the society should belong to the non-singleton component. Finally, the ordered conditions are easily verified. This completes the proof of strict equilibria with some non-singleton components. Hence, the proof of the first part of the proposition follows.

Second part: I now prove the converse. Let $g$ be a wheel and set $c_{i}<V$ for any $i \in N$. I note that given $c_{i}<V$ any wheel is ordered (no player wants to switch) and that each player is playing his unique best response. Therefore $g$ is a strict equilibrium. The same set of restrictions in the costs of linking imply that the flower network with player 1 the center is a strict equilibrium. Finally, suppose that $g$ is a wheel with a periphery-sponsored star with player 1 the center. Set $c_{i}<V$ for any $i \in C(g)$, while $c_{j}>n V$ for any $j \notin C(g)$. It follows that $g$ is ordered and it is a strict equilibrium. The same conditions applies to the flower network with a periphery sponsored star with player 1 the center. This completes the proof of the second part of the proposition.

The proof of the proposition in now completed.

I conclude by providing the characterization of strict equilibria in which each component is composed of a single player. I first introduce some notations. A generalized periphery sponsored star with center $j$ is an unconnected architecture where each component is a singleton and where there exists a player, the center $j$, such that 
$g_{i, j}=1$ for some (or all) $i \in N$ and the remaining players, say $\left\{j_{1}, j_{2}, \ldots, j_{m}\right\} \subseteq N$, are arranged in the following way $\left\{g_{j, j_{m}}=g_{j_{m}, j_{m-1}}=\ldots=g_{j_{2}, j_{1}}=1\right\}$. Note that if $\left\{j_{1}, \ldots, j_{m}\right\}=N$ then $g$ is a line where the information flow from $j_{m}$ to $j$, from $j_{m-1}$ to $j_{m}$ and so on. While if $\left\{j_{1}, \ldots, j_{m}\right\}$ is empty then $g$ is a periphery-sponsored star with player $j$ the center. The next definition defines the ordered condition for a generalized-periphery sponsored star network.

Definition 3. A generalized periphery-sponsored star network $g$ is ordered if $\forall$ $g_{j^{\prime}, j^{\prime \prime}}=1$ then $c_{j^{\prime \prime}}-c_{y}<d_{j^{\prime \prime}, y}(g) V, \forall y$ accessed by $j^{\prime}$ via the link $g_{j^{\prime}, j^{\prime \prime}}=1$.*********

Proposition 4.2. Let (3)-(4) be satisfied and let $g$ be a strict equilibrium in which each component is composed of a single player. Then $g$ is either the empty network or the ordered generalized periphery sponsored-star network where $g_{1, i}=0$ for any $i \in N$. Conversely, any such network is a strict equilibrium for some $\left\{c_{i}, V\right\}$.

Proof. The first part of Proposition 4.2 is based on two Lemmas.

Lemma 4.5: Let $g$ be an unconnected strict equilibrium. If $g_{i, j}=1$ and $g_{k, j}=1$ then $i$ and $k$ do not receive any link, i.e. $g_{j^{\prime}, i}=g_{j^{\prime}, k}=0 \forall j^{\prime} \in N$.

Proof: For a contradiction, suppose first that $g_{j^{\prime}, k}=1$ for some $j^{\prime} \in N$. Since $g$ is minimal $j^{\prime} \neq i$; since $g$ is unconnected $j^{\prime} \neq j$ and is different from any player $k^{\prime}$ such that there exists $j \stackrel{g}{\rightarrow} k^{\prime}$ in $g$. Since $g$ is strict Nash, the link $g_{i, j}=1$ implies that $c_{k}-c_{i}>V$, and the link $g_{j^{\prime}, k}=1$ implies that $c_{k}-c_{j}<V$. This is a contradiction. The same applies to the case where $g_{j^{\prime}, i}=1$. This completes the proof of the Lemma.

Lemma 4.6 Let $g$ be a non-empty unconnected equilibrium where each component is a singleton. Then each player promotes at most one link.

Proof: Suppose not, i.e. there exists a player $j$ such that $g_{j, i}=g_{j, k}=1$ for some distinct $k$ and $i$. We first note that if $g_{j, i}=1$, then player $j$ accesses via the link with player $i$ some player, say $i^{\prime}$, such that $g_{i^{\prime}, i^{\prime \prime}}=0$ for any $i^{\prime \prime} \in N$. To see this note that if $g_{i, i_{1}}=0$ for any $i_{1} \in N$, the proof trivially follows. Moreover, if $g_{i, i_{1}}=1$, then $i_{1} \neq j$; for otherwise $i$ and $j$ would be part of a non-singleton component. Iterating the argument and noting that set of player is finite, the claim follows. Second, this implies that if player $j$ is linked with $i$ and $k$, then there exist $\left\{g_{j, i}=g_{i, i_{1}}=\ldots=g_{i_{n}, i^{\prime}}=1\right\}$ and $\left\{g_{j, k}=g_{k, k_{1}}=\ldots=g_{k_{n}, k^{\prime}}=1\right\}$, where $g_{i^{\prime}, s}=g_{k^{\prime}, s}=0$ for any $s \in N$. It is easy to see that if $k^{\prime}=i^{\prime}$ then Lemma 4.5 is violated. Furthermore, if $k^{\prime} \neq i^{\prime}$ since $g$ is a strict Nash equilibrium, player $k_{n}$ strictly gains by forming a link with player $i^{\prime}$. This constitutes a contradiction. Hence, the proof follows.

It is easy to see that Lemma 4.5 and 4.6 implies that a strict equilibrium where each component is a singleton has a generalized periphery-sponsored star. Moreover, if the ordered condition does not hold than some player has an incentive to deviate, which 
contradicts the fact that $g$ is a strict Nash equilibrium. Finally, we note that the agent which does not promote any link is always the player with the lowest access cost in the whole society, i.e. player 1. Suppose, for a contradiction, that player $k$ does not promote any link. Since there exists a player, say $j$, such that $g_{j, k}=1$, it follows that $c_{k}<V$, but then also $c_{1}<V$ and therefore player $k$ strictly gains by creating a new link with player 1 . This completes the proof of the first part of the proposition

Second Part: Let $g$ be the empty network and let $c_{i}>V$ for any $i \in N$; it is trivial to see that the empty network is a strict equilibrium. I finally consider the case where $g$ has a generalized periphery-sponsored star architecture where player 1 is the agent which does not promote any link. Set the following conditions on the costs of linking: (i) $c_{1}<V$, (ii) for any $i \in N$ such that $g_{i, j}=1$ then $c_{i}>n V$, (iii) $m V<c_{j}<(m+1) V$ and (iv) $(x-1) V<c_{j_{x}}<x V$, for any $x=2, \ldots, m$. These conditions imply that: one, each agent who promotes a link obtain a strictly positive utility, two no agent wants to form an additional link. Therefore, given these conditions, whenever $g$ is ordered $g$ is also a strict equilibrium. ${ }^{17}$. This completes the proof of the second part of the proposition.

The proof of Proposition 4.2 is complete.

\section{References}

[1] R. Aumann and R. Myerson (1989), Endogenous formation of links between players and coalitions: an application of the Shapley Value, in A. Roth (ed) The Shapley Value. Cambridge University Press. Cambridge.

[2] V. Bala and S. Goyal (2000a), A Non-cooperative Model of Network Formation, Econometrica, 68, 1181-1229.

[3] K. Basu and J.W. Weibull (1991), Strategy subsets closed under rational behavior, Economics Letters, 36, 141-146.

[4] R. Burt (1992), Structural Holes: The social structure of competition. Harvard University Press, Cambridge, MA.

[5] J. Coleman (1966), Medical Innovation: A diffusion study. Bobbs-Merrill, New York.

\footnotetext{
${ }^{17}$ Note that if in the generalized periphery sponsored star players are arranged as follow: $g_{y, x}=1$ for any $y>x$ and $\left\{g_{x, x-1}=g_{x-1, x-2}=\ldots=g_{2,1}=1\right\}$, then $g$ is always ordered whenever $c_{h}-c_{h-1}<V$ for any $h=1, \ldots, x$. This condition is consistent with conditions (i)-(iii).
} 
[6] B. Dutta and M. Jackson (2000), The stability and efficiency of directed communication networks, Review of Economic Design, 5, 251-272.

[7] A. Falk and M. Kosfeld (2003), It's all about connections: Evidence on network formation, mimeo, University of Zurich.

[8] S. Goyal (1993), Sustainable Communication Networks, Tinbergen Institute Discussion paper, 93-250

[9] A. Galeotti, S. Goyal, J. Kamphorst (2003), Network formation with heterogeneous players, mimeo, Tinbergen Institute.

[10] M. Granovetter (1974), Getting a job: A study of contacts and careers. University of Chicago Press, Chicago.

[11] H. Haller and S. Sarangi (2001), Nash networks with heterogeneous players, mimeo, VPI.

[12] M. Jackson and A. Wolinsky (1996), A Strategic Model of Social and Economic Networks, Journal of Economic Theory, 71, 44-74.

[13] C. Johnson and R.P. Gilles (2000), Spatial Social Networks, Review of Economic Design, 5, 3, 273-301.

[14] C. Kim and K.C. Wong (2003), Network Formation and Stable Equilibrium, mimeo.

[15] M. McBride (2002), Position-specific Information in Social Networks, University of California.

[16] J. Montgomery (1991), Social networks and labor market outcomes: toward an economic analysis, American Economic Review, 81, 5, 1408-1418.

[17] M. Slikker and A. van den Nouweland (2001a), A one-stage model of link formation and payoff division, Games and Economic Behavior, 34, 153-175.

[18] A. Watts (2001a), A Dynamic Model of Network Formation, Games and Economic Behavior, 34, 331-341.

[19] A. Watts (2001b), Non-Myopic Formation of Circle Networks, Economics Letters, 74, 272-282. 


\section{Figures:}

Figure 1

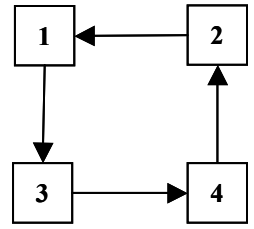

Wheel

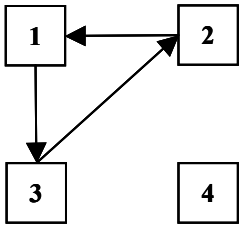

Wheel with singletons

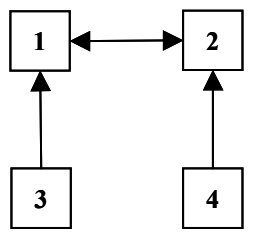

Wheel with local centersponsored stars

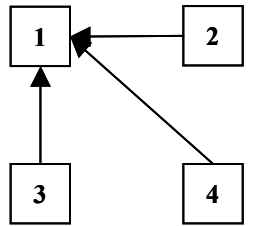

Center-sponsored star

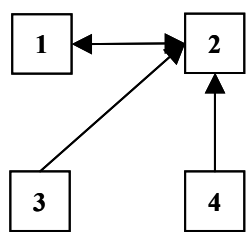

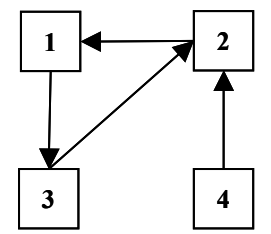

4

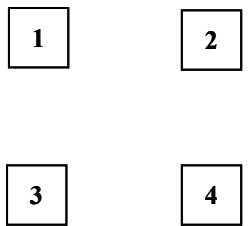

Empty network 
Figure 2

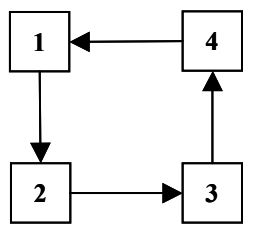

Wheel
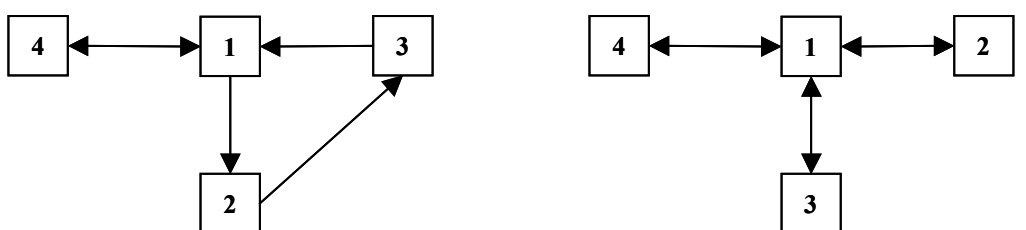

Flower with center 1
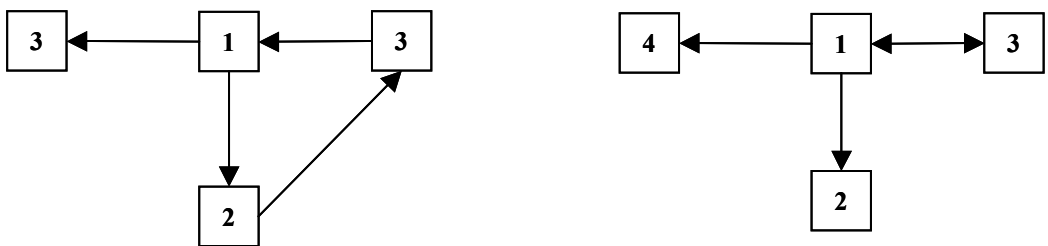

Wheel with a local periphery-

sponsored star with center 1

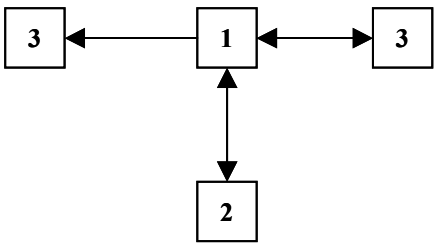

Flower with a local peripherysponsored star with center 1 
Figure 3

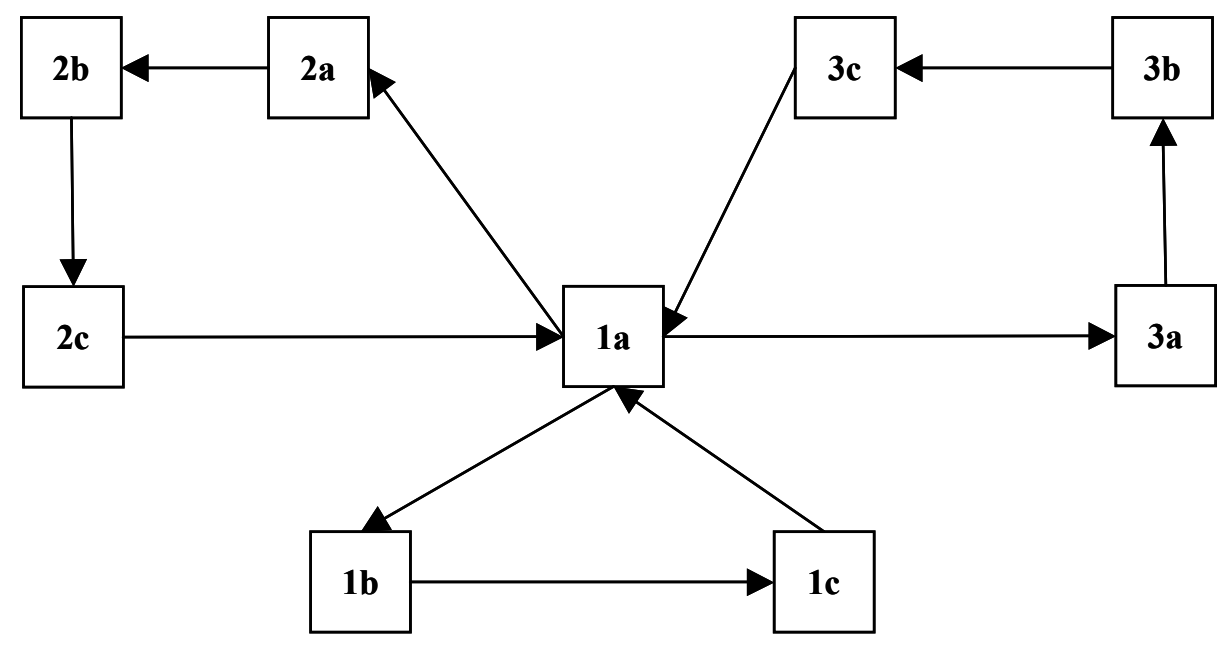

